TAO, Vol. 16, No. 2, 315-329, June 2005

\title{
Array Observations for Narrow-Band Background Noises in the Hualien Area and Their Seismological Implications
}

\author{
Ya-Chuan Lai $^{1, *}$, Bor-Shouh Huang ${ }^{2}$, Hong-Yuan Yen ${ }^{1,2}$, Kou-Cheng Chen ${ }^{2}$, \\ Yi-Ling Huang ${ }^{1}$, Yi-Ru Chen ${ }^{1}$ and Juen-Shi Jiang ${ }^{1,3}$
}

(Manuscript received 9 October 2003, in final form 25 January 2005)

\begin{abstract}
Seismic observations in the Hualien area have been greatly disturbed by narrow-band background noise. Thus, the automatic earthquake trigging system of the Taiwan regional seismic network has been equally affected by the same background signals, the origin of which is still controversial. Herein, array measurements were conducted to account for this seismic noise and to recognize its seismic wave propagation properties. Five temporary arrays were designed to observe this background noise. Each array consisted of 15 three-component seismometers spaced from 100 to 300 meters apart. Observed data shows that a notable band limit background noise, in the frequency range of 1.3 to $1.7 \mathrm{~Hz}$, was identified from its seismic power spectrum with strong separated peaks at $1.3 \mathrm{~Hz}, 1.4 \mathrm{~Hz}$ and $1.7 \mathrm{~Hz}$. The origins and characteristics of the background noises were investigated using frequency-wavenumber analyses and waveform stacking techniques. Analyzed results suggested that the feasible sources of the narrow-band signals were located near the southwest boundary of the survey area. Based on particle motion analysis, the narrow-band seismic signals were identified as major Rayleigh wave type ground motions. In this survey area, microtremors were generated from multiple origins. However, the major signal sources remain stable in the southwest. Those signals were also examined with regard to persistence over time from a permanent seismic station leading to the inference that the source of that background noise was industrial in origin.
\end{abstract}

\footnotetext{
${ }^{1}$ Institute of Geophysics, National Central University, Chung-Li, Taiwan, ROC

2 Institute of Earth Sciences, Academia Sinica, Taipei, Taiwan, ROC

${ }^{3}$ Seismology Center, Central Weather Bureau, Taiwan, ROC

* Corresponding author address: Ms. Ya-Chuan Lai, Institute of Geophysics, National Central University, Chung-Li, Taiwan, ROC; E-mail: yachuan@earth.sinica.edu.tw
} 
(Key words: Seismic array, Frequency-wavenumber analysis, Microtremors)

\section{INTRODUCTION}

The Hualien area is an important site for earthquake observation in Taiwan where short period seismometers, strong motion arrays and broadband instruments are installed. However, there is a narrow band background noise which usually disturbs earthquake observations in the Hualien area (Fig. 1). Seismic observation in Hualien is greatly affected by this noise, especially for the automatic event detection of the Central Weather Bureau Seismic Network (CWBSN). Miss picking of first-arrival time is usually found at Station TWD (a CWBSN station located at northern Hualien) as a result of this background noise (C. C. Liu, personal communication). However, the source of this noise is still unknown. To improve data quality of seismic observation in this area, the origin of this noise and its characteristics need to be identified. However, at present there is a difficulty in analyzing the background noise using available data because the noise observed at Station TWD is in near harmonic oscillation with an event, thus necessitating an array observation.

A seismic array is a set of seismographs distributed over an area of the Earth's surface with spacing narrow enough that signal waveform may be correlated between adjacent seismographs. Array observations are useful when studying the detailed characteristics of wave field propagation across an array (Aki and Richards 1980). Array studies concerned with background seismic noises (microtremors) have been reported by many authors (Capon 1969; Haubrich and MaCamy 1969). Asten and Henstridge (1984) reviewed possible mechanisms of generating sources and propagation modes of microtremors and concluded that cultural sources, coastal effects, ocean storms, atmospheric loading and artificial noises (vehicles and railway) were the primary types of microtremor origins which can be identified within a frequency range of 0.5 to $10 \mathrm{~Hz}$. Walker et al. (1964) studied spectra up to a few Hertz and found sets of extremely narrow-band signals which persisted over days. The spectral lines started and stopped suddenly, in opposition to the continuity of seismic signals and suggested an artificial origin. Douze (1967) also found signals at a stable frequency did not decay in depth from borehole data, again suggesting an industrial origin of the noises. The nature of such signals at 15 stations distributed across Germany was studied by Bokelmann and Baisch (1999) where they observed several persistent spectral lines most prominent with a frequency near $2.083 \mathrm{~Hz}$. Almendros et al. (2002) tracked the source motion using seismic arrays in Hawaii because the arrays can sample ground motion in both time and space. Their investigation of the observations revealed that an artificial seismic source, in this case heavy vehicles, was responsible for the activity. Most of those sources generated Rayleigh waves and part of the sources generated the $\mathrm{P}$ and Love waves.

The microtremors in the Hualien area, in general, can be attributed from neither a single mode nor a single direction of propagation. Extraction of useful information from this narrow -band background noise requires an array of seismometers combined with suitable digital data processing techniques. To understand the fundamental propagation properties and source of this noise, a field experiment for microtremors survey was proposed. The frequency- 
wavenumber (F-K) analysis (Capon 1969) which estimated the likeness of signals on frequency $(\mathrm{F})$ and a wavenumber $(\mathrm{K})$ domain was employed in this study. Results of this study showed that background noise abounds in low frequency band-limit signals, from $1.3 \mathrm{~Hz}$ to $1.7 \mathrm{~Hz}$ as observed at all array stations. The signals showed strong peaks near $1.3 \mathrm{~Hz}, 1.4 \mathrm{~Hz}$ and $1.7 \mathrm{~Hz}$ in its power spectra and three different frequency picks indicate consistent, low phase velocity seismic waves across the survey area with incidents originating from a southwest direction. Using a combination of F-K and waveform stacking analyses, source positions were identified wherein the detected signals suggest industrial origins induced by synchronous machine activity.

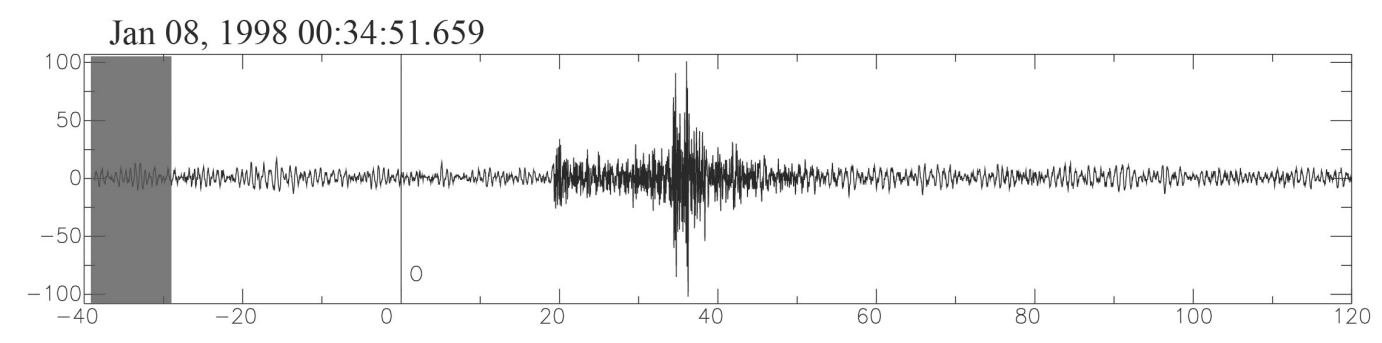

Jan 11, 1998 04:50:13.020

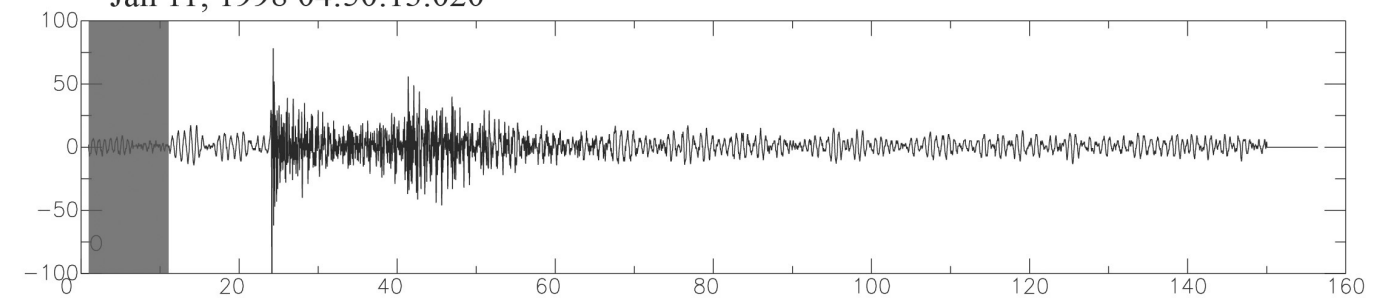

Jan 13, 1998 12:43:52.959

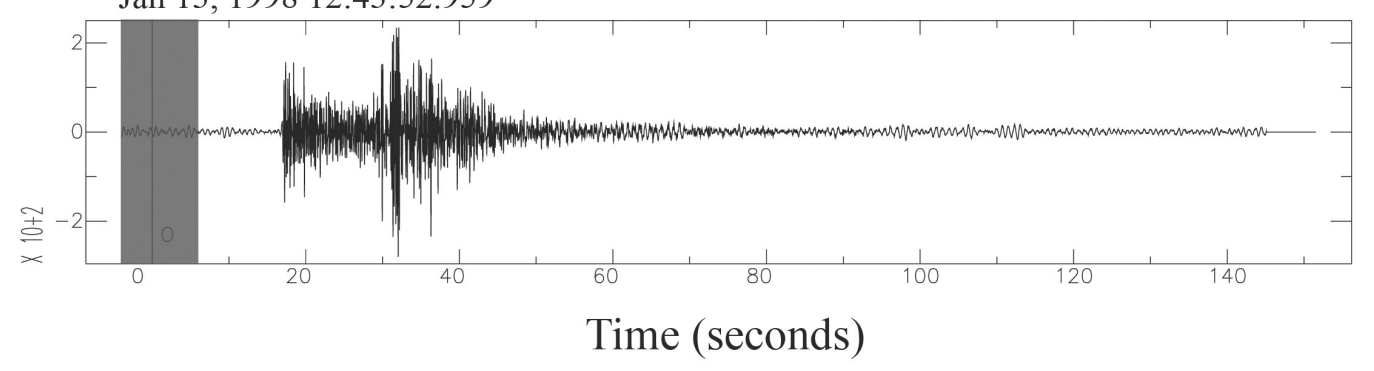

Fig. 1. The selected vertical component velocity seismograms from Station TWD wherein background noise persists to cover the arrival of a microearthquake. The first ten-second section, marked as the gray window, was selected for estimating the power spectrum to study its temporal variation and will be discussed in detail in the text. In each panel, the origin time (marked by vertical bar with zero) for each event is showed in the top and the amplitude is in terms of digital counts. 


\section{OBSERVATION}

In order to understand the nature and behavior of the narrow-band signals, dense smallaperture arrays were operated in the northern Hualien area by the Institute of Earth Sciences, Academia Sinica in April 1991. The intensive arrays in the region surrounding Station TWD designed for this experiment are shown in Fig. 2. The survey area lies between the coastal line and mountain area. The surface of this area is covered by sediments. The array measurements

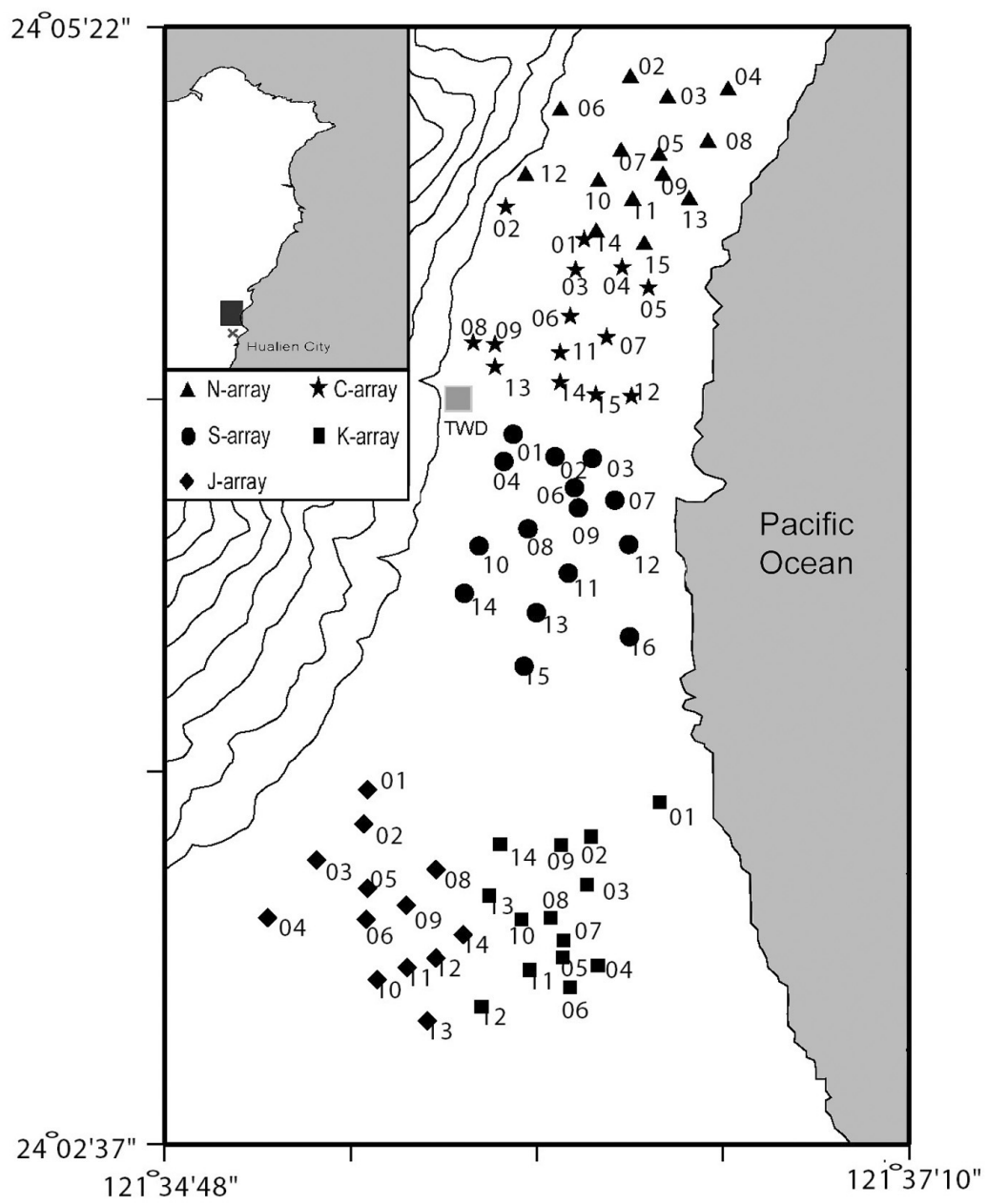

Fig. 2. Map of the survey area and temporary stations operated in the northern Hualien area. Five arrays with different symbols consisting of seventy stations are called the N, C, S, K and J-arrays from north to south. The solid large square indicated the location of permanent TWD seismic station operated by the CWBSN. 
were deployed as five subnets because of the limited availability of instruments. Three-component short-period seismometers (Mark Product L-4C, $\mathrm{T}_{0}=1 \mathrm{~s}$ ) were installed at all stations during this experiment. The recorded data was sampled at $100 \mathrm{~Hz}$. Most of stations were on a plain area with a few stations on the western boundary of the array in a foothill area. Elevation differences between array stations were less than $20 \mathrm{~m}$. The survey area has been occupied by five group seismometers called the N, C, S, K and J-arrays, from north to south (Fig. 2). The station distances were at $200 \mathrm{~m}$ on average. Instrument recording times were provided by internal clocks calibrated by a master clock. There were three observed time windows on each station. Each window had a three minute window of continuous recording.

Figure 3a shows some examples of the microtremors, no clear traveling phases were identified from that data. The specific signals were persistent in all records. In addition, some highfrequency transient noises were found during array observation. Such noises marked as "Phase-V" in Fig. $3 b$ have been identified as vehicle generated noises garnered from, field reports during noise observation and were discounted in this study. In order to understand the characteristics of the persistent signals we estimated the power spectrum of each observed time window data individually for all stations by Fourier transform. Stacking power spectra of three time window records was employed to stress the frequency characteristics at each station. The stacking power spectrum of all stations shows the notable band-limit spectrum features from 1.3 to 1.7 $\mathrm{Hz}$ and strong peaks near $1.3 \mathrm{~Hz}, 1.4 \mathrm{~Hz}$, and $1.7 \mathrm{~Hz}$, respectively. Therefore a stack of amplitude spectrum of all stations in each subnet would reveal prominent properties in the experiment region, with narrow band from $1.3 \mathrm{~Hz}$ to $1.7 \mathrm{~Hz}$ and strong peaks near $1.3 \mathrm{~Hz}, 1.4 \mathrm{~Hz}$, and $1.7 \mathrm{~Hz}$ as shown in Fig. 4. With regard to the seismic spectra, other minor peaks were found at the double frequencies of these major peaks, $2.6 \mathrm{~Hz}, 2.8 \mathrm{~Hz}$ and $3.4 \mathrm{~Hz}$ wherein the signals varied slightly. To investigate the noise origin in detail, array methods have been proposed to analyze those data.

\section{METHOD}

The ground motion of a two-dimensional plane, represented as $v(x, y, t)$, may be represented by its frequency $(\omega)$ and wavenumber $(k)$ components using the three dimensional Fourier transform as:

$$
V\left(\omega, k_{x}, k_{y}\right)=\iiint v(t, x, y) \exp \left[-i\left(\omega t+k_{x} x+k_{y} y\right)\right] d t d x d y .
$$

In which, in terms of the azimuth angle $\theta, k_{x}=|k| \sin \theta$ and $k_{y}=|k| \cos \theta$ are two decomposed horizontal components of wavenumber $(k)$. The spatiality of a seismic array, however finite and discrete, allows the estimation of the $V\left(\omega, k_{x}, k_{y}\right)$ of Equation (1) and thus an estimate of the wavenumber and its slowness in propagating signals and noises. This versatility of seismic arrays is due to the fact that they sample the ground motion in both time and space, thus allowing a separation of different wave components in the wave field and provide an estimation of the phase velocity and the approach direction of these waves. To estimate the $\omega-k$ spectra, two methods were developed, the Beam-forming Method (BFM) (Lacoss et al. 


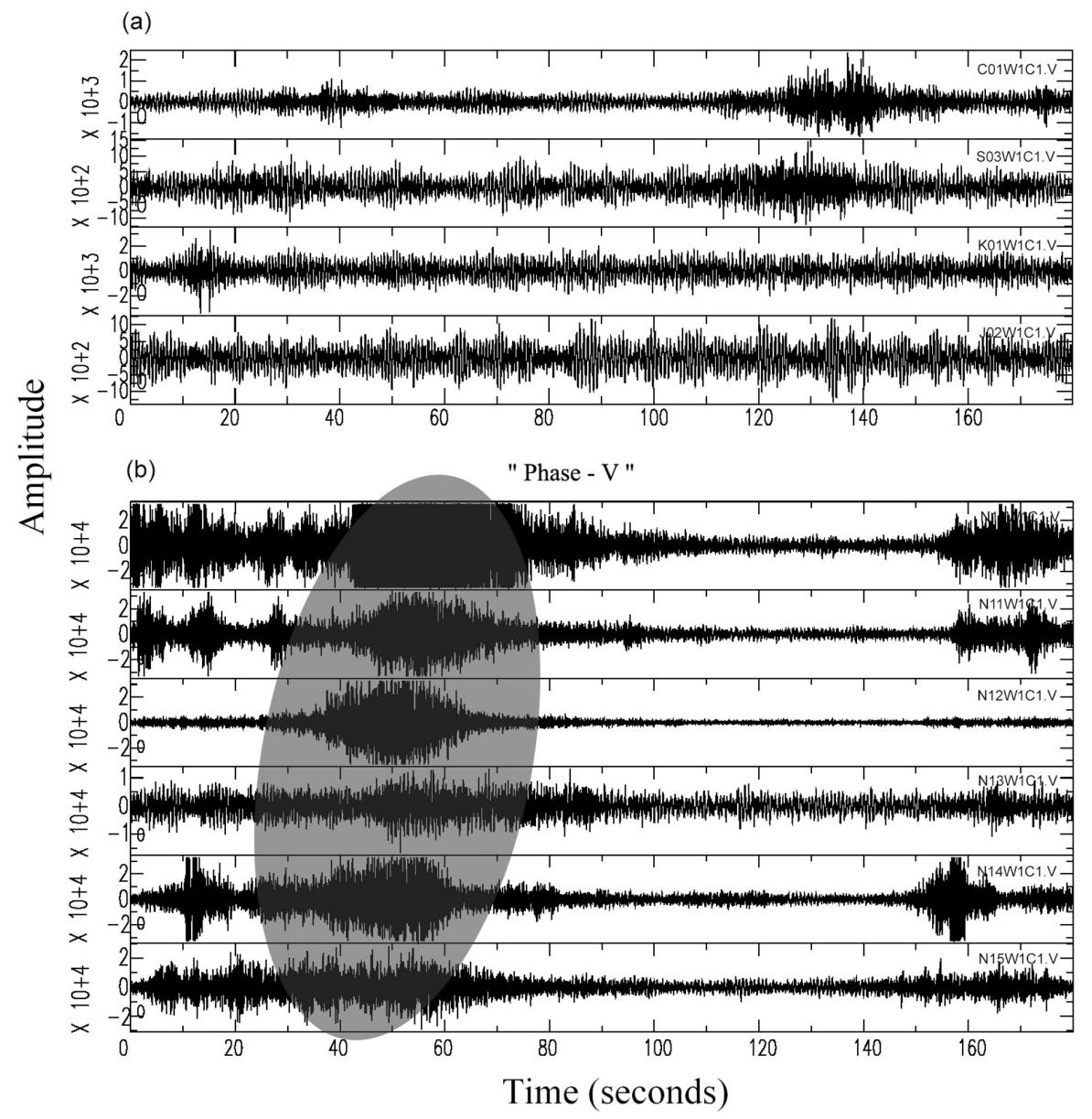

Fig. 3. (a) Selected vertical velocity seismograms recorded from the different subnet of this array experiment. The relative time with the different initial time of each subnet is adopted for display. (b) Vertical array seismograms recorded by the $\mathrm{N}$-array. The ellipsoid marked "Phase- $\mathrm{V}$ " indicated signals induced by a train across this seismic array. The vertical axis on each panel is in terms of digital counts of the seismogram.

1969) and Maximum-Likelihood Method (MLM) (Capon 1969). For a more detailed discussion of both methods see Huang and Yeh (1990). Generally, the MLM is the better method for processing two dimensional array data to achieve high resolution in the presence of multi-path interference and is therefore, most suitable for background noise studies for use in this study.

Based on the estimated phase velocity of background noises from the F-K analyses, the array signals can be stacked to determine its source location. Herein, a simple scheme is em- 


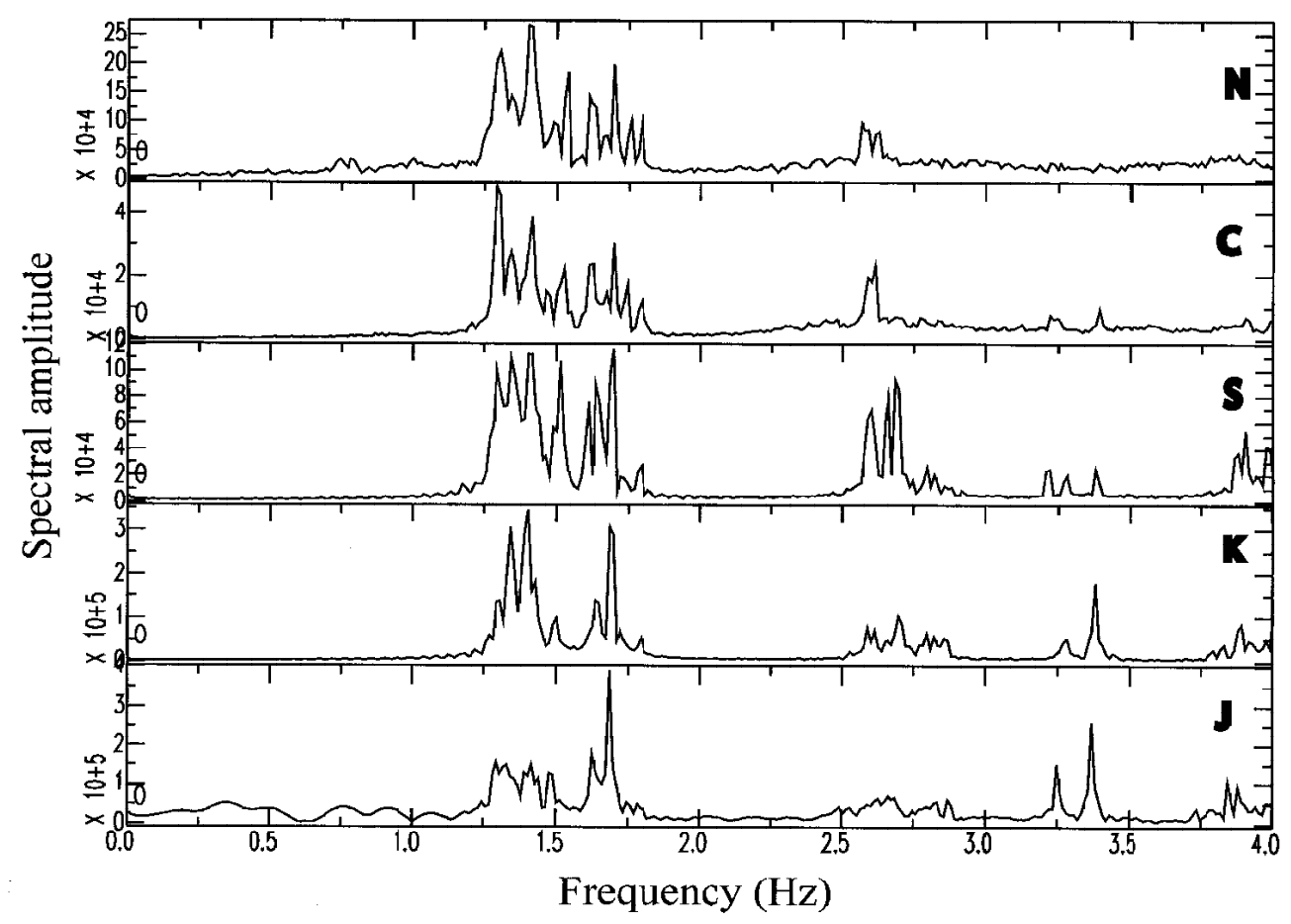

Fig. 4. The stacking power spectra with respect to three time windows for the vertical component seismograms for all stations at the N, C, S, K and Jarrays (from top to bottom). The estimated spectral amplitudes are in terms of digital counts.

ployed to process the array data to locate its possible source locations. This method is expressed as:

$$
B(x, y, t)=\sum_{j}\left[\sum_{i=1}^{N} A_{i, j+i t}\right] .
$$

$N$ is the number of the stations, and $i$ means the $i t h$ station; $j$ is the time window to stack. The connotation it is the theoretical travel time $t$ of seismic wave from source located on $(x, y)$ to the $i t h$ station and $A_{i, j+i t}$ is the amplitude of the ith station in $j+i t$ time. By this method we can calculate the waveform stacking value $B$ for each location in the survey area to estimate the possible source locations. In this study we used 2-D grid points with a spacing of $100 \mathrm{~m}$. The stacking value at a particular grid point is related to the magnitude of coherency there. Herein, the image of possible sources is determined from its highest stacking value $B(x, y, t)$ with respect to time. 


\section{RESULTS}

The analyses for each array are expressed in terms of F-K spectral response of the signals at specified times, about thirty seconds in the three-minute records, with no microearthquake or transient noise (Fig. 5). These seismic signals are characterized in the plane wave-front approximation and in terms of the apparent slowness vectors. The magnitude of the slowness vector is used to determine the apparent velocities of propagation signals. The vector direction of slowness is considered as the wave propagation azimuth, usually measured clockwise from the north. Figure 5a shows the F-K responses of the vertical component seismograms of the N, $\mathrm{C}$ and $\mathrm{S}$ arrays from filtered narrow frequency bands of $1.30 \pm 0.1 \mathrm{~Hz}, 1.4 \pm 0.1 \mathrm{~Hz}$ and $1.7 \pm 0.1 \mathrm{~Hz}$. In this figure the contours show the seismic energy of signals, and the center of dense contours is the slowness vector of coherent signals. The F-K spectral responses of the signals at 1.3,1.4, and $1.7 \mathrm{~Hz}$ show a similar pattern and imply the same origins in the southwest direction.

The F-K spectral responses suggest more than one origin and vary with arrays slightly (Fig. 5). For the $1.3 \mathrm{~Hz}$ signals, the spectral response for $\mathrm{N}$-array suggests dominant energy with a back-azimuth of 241 degrees and phase velocity of about $1.03 \mathrm{~km} \mathrm{~s}^{-1}$. The result for Carray suggests some arrivals with a back-azimuth of 252 degrees and phase velocity of about $0.84 \mathrm{~km} \mathrm{~s}^{-1}$, and other arrivals show a back-azimuth of 112 degrees and phase velocity of about $0.41 \mathrm{~km} \mathrm{~s}^{-1}$. The result of S-array shows a similar energy distribution as the $\mathrm{N}$-array in general with the predominant energy incident from the southwest (Fig. 5a). However, spectral responses are more complicated in the $\mathrm{J}$ and $\mathrm{K}$-arrays (Fig. 5b). Although the energy distributions for signals at $1.3 \mathrm{~Hz}$ are varied with the arrays, the slowness plots generally show some signals incident from the east and the others from the west. Hence, more than one source is suggested.

The rough source locations near the J-array can be determined based on the estimation of the intersections of all the back-azimuths provided by the prominent energy in the F-K analyses. This simple geometrical method was applied by Almendros et al. (2000) to determine the sources of volcano tremors. Results of this study indicated that the sources approached from the southwest direction of this area. The sources within or near the $\mathrm{K}$ and J-arrays may be the reason why the signals are much more incoherent and show a complexity in its frequencywavenumber spectral responses (Fig. 5b).

Employing the waveform-stacking method we can obtain a more explicit source position. For calculating the stacking value of Equation (2), we assumed the seismic wave propagation in the horizontal plane. We changed the source location with a $0.1 \mathrm{~km}$ shift both in longitude and latitude and accounted for all travel time corrections. Estimating the time correction of each station for all possible source locations, the phase velocity provided from the F-K analyses for each array was taken as the average propagation speed. The stacking value would be higher when the assumed source is close to its true location. In this study we defined the region with a stacking value greater than $90 \%$ of its maximum as possible source locations. Figure 6 shows the stacked image for $1.7 \mathrm{~Hz}$ signals in first time window, and a notably high value region is near the J-array. Moreover, such regions were obtained for all data in other time windows. Similar processed results from other frequency bands showed a consentient pattern 
(a)

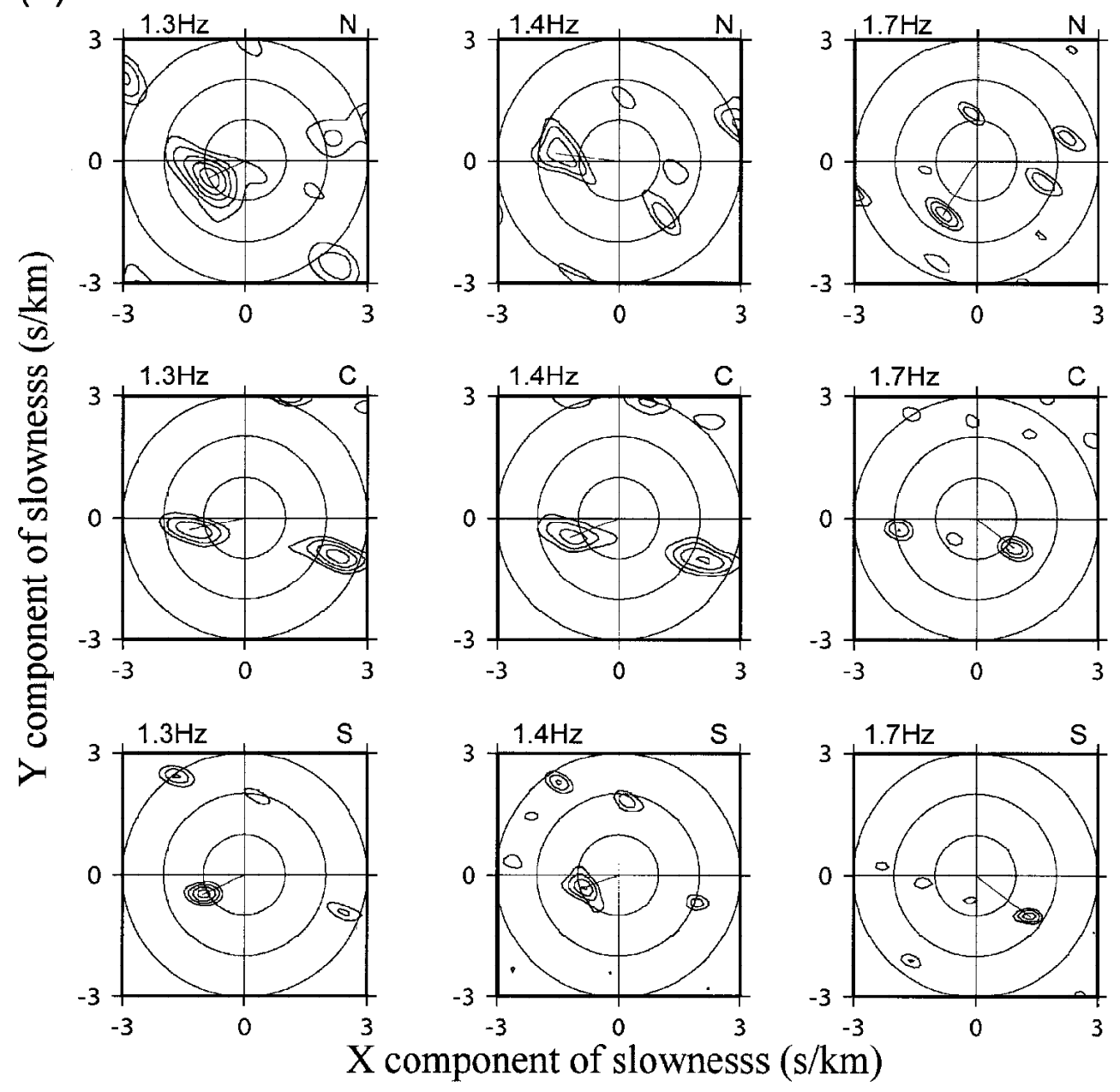

Fig. 5. (a) The F-K spectral responses of vertical component seismograms at $1.3 \mathrm{~Hz}, 1.4 \mathrm{~Hz}$ and $1.7 \mathrm{~Hz}$, individually, for the N, C and S-arrays. (b) The F-K spectral responses of vertical component seismograms at $1.3 \mathrm{~Hz}, 1.4 \mathrm{~Hz}$ and $1.7 \mathrm{~Hz}$, individually, for the $\mathrm{J}$ and $\mathrm{K}$-arrays. The average frequency of signals for each array is indicated at the top of each plot. On each panel, the contour represents signal power and the highest amplitude represents the arrivals with the primary seismic energy across the array. The lines from origins to the centers of the denser contours show the slowness vectors for N, C, and S-arrays but not for $\mathrm{K}$ and $\mathrm{J}$ arrays. The three circles from large to small represent the constant slowness of the arrivals, $3 \mathrm{~s} \mathrm{~km}^{-1}, 2 \mathrm{~s} \mathrm{~km}^{-1}$ and $1 \mathrm{~s} \mathrm{~km}^{-1}$, respectively. 


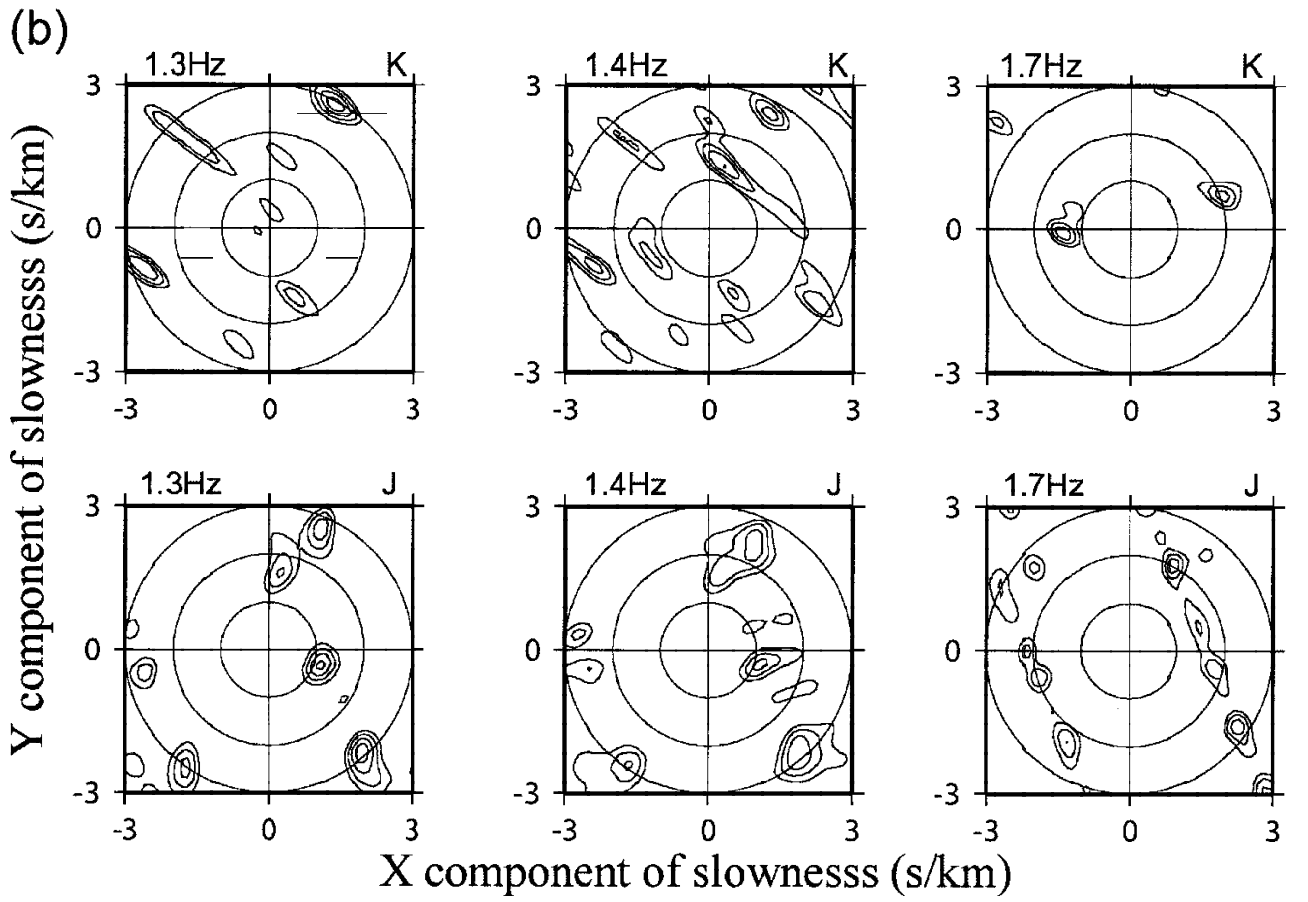

Fig. 5. Continued.

for the high stacking value near the southwest border of the survey area.

To know the wave type of the microtremors, the recorded three-component time traces have been employed. In this study, the particle-motions of the narrow-band signals have been investigated for each station in the K and S-arrays. Assuming the approaching seismic signals originated from a southwest direction in this survey area, time traces were rotated accordingly. The particle-motions obtained from most of the stations show elliptical retrogressions and suggest the major energy propagated as Rayleigh wave (Fig. 7). However, in some cases, a visible energy was also found on the tangential component seismograms.

To identify the origin of those narrow-band background noises at Station TWD independently, we examined the pre-event background noises from seismic records (the gray windows in Fig. 1). We analyzed seismograms of Station TWD from all events recorded by the CWBSN within a selected time window (120 days) from 1 Jan. 1998. One record per day, at least, was selected from the CWBSN waveform data bank. Each selected vertical component seismogram of Station TWD was cut before the time of P-wave arrival as daily background noise for further analysis in this study. Each time trace was transformed to its amplitude-frequency spectrum. All of these spectra were then displayed with respect to its event occurrence times as shown in Fig. 8 and revealed some signals persisted with time in the amplitude spectra of the background signals. The same as array observations in this study, the 

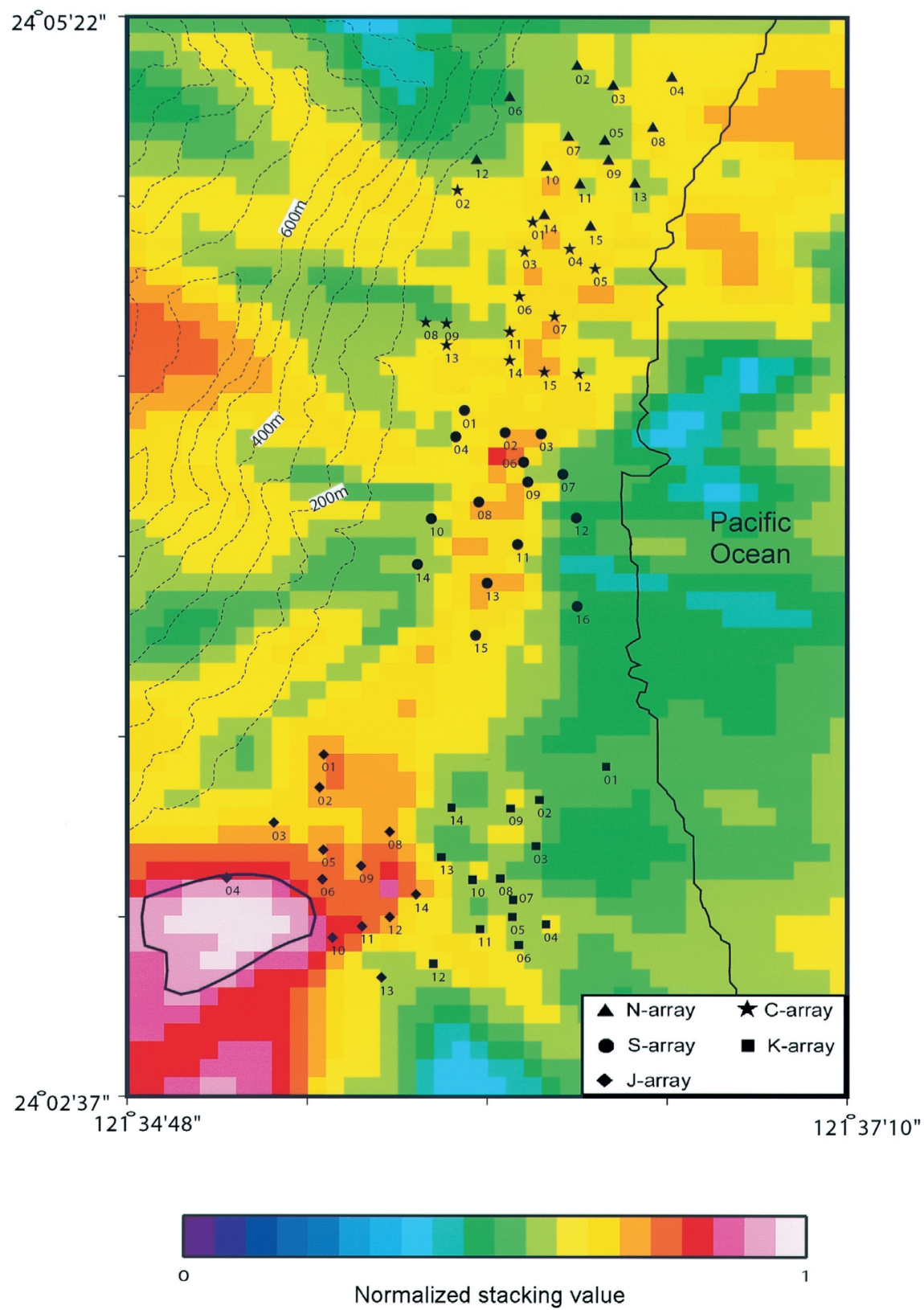

Fig. 6. The stacked image constructed from all recorded narrow-band vertical component seismograms near $1.7 \mathrm{~Hz}$. The beam value of each grid point is normalized with the maximum and is represented by colors as shown in scale bar. The region with beam value larger than $90 \%$ of its maximum value is indicated by the solid line. 

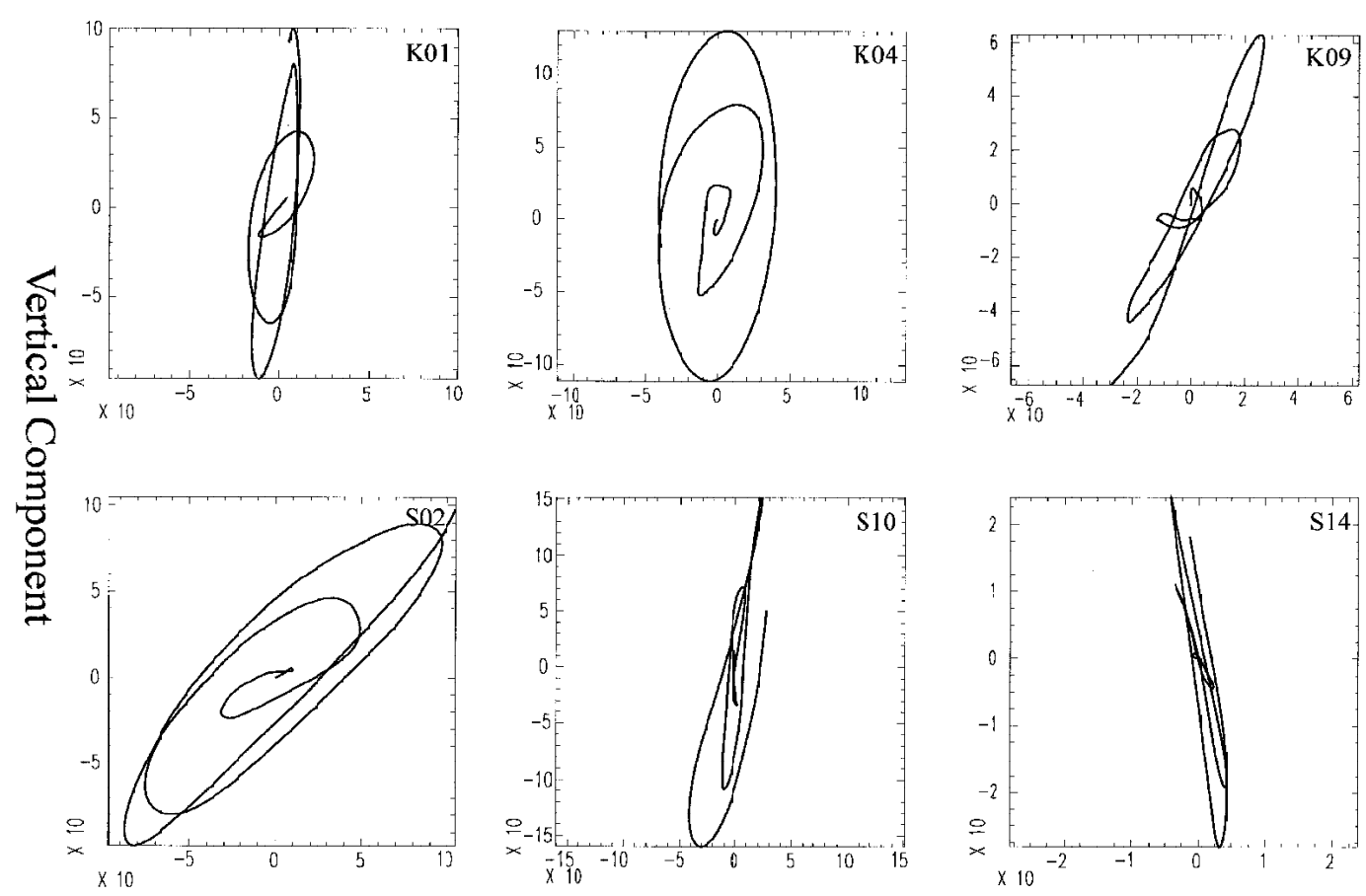

\section{Radial Component}

Fig. 7. The in-plane particle-motion determined from filtered seismograms of some stations of the $\mathrm{K}$ and $\mathrm{S}$-arrays. The station id is marked in the upper right corner on the panel and the position of which is shown in the Fig. 2. In each plot, the initial point is taken as the zero-point and the trajectory shows an elliptical retrogression suggesting the narrow-band energy propagates as Rayleigh wave. The amplitude is in terms of digital counts.

high amplitude of the background signals at $1.1 \mathrm{~Hz} 1.7 \mathrm{~Hz}$ were clearly observed at Station TWD. However, two short noise-quiet windows of the background signals were found during the analyzed 120-day time window. A detailed examination of the earthquake catalog indicated that one quiet period was related to a two day no event data gap and the other to a one week Chinese New Year vacation. Thus, during the New Year vacation, no clear narrow-band background noises were found from recorded seismograms at Station TWD. Herein, this signal gap is thus interpreted as the shutdown of some artificial noise sources during the Chinese New Year vacation. 

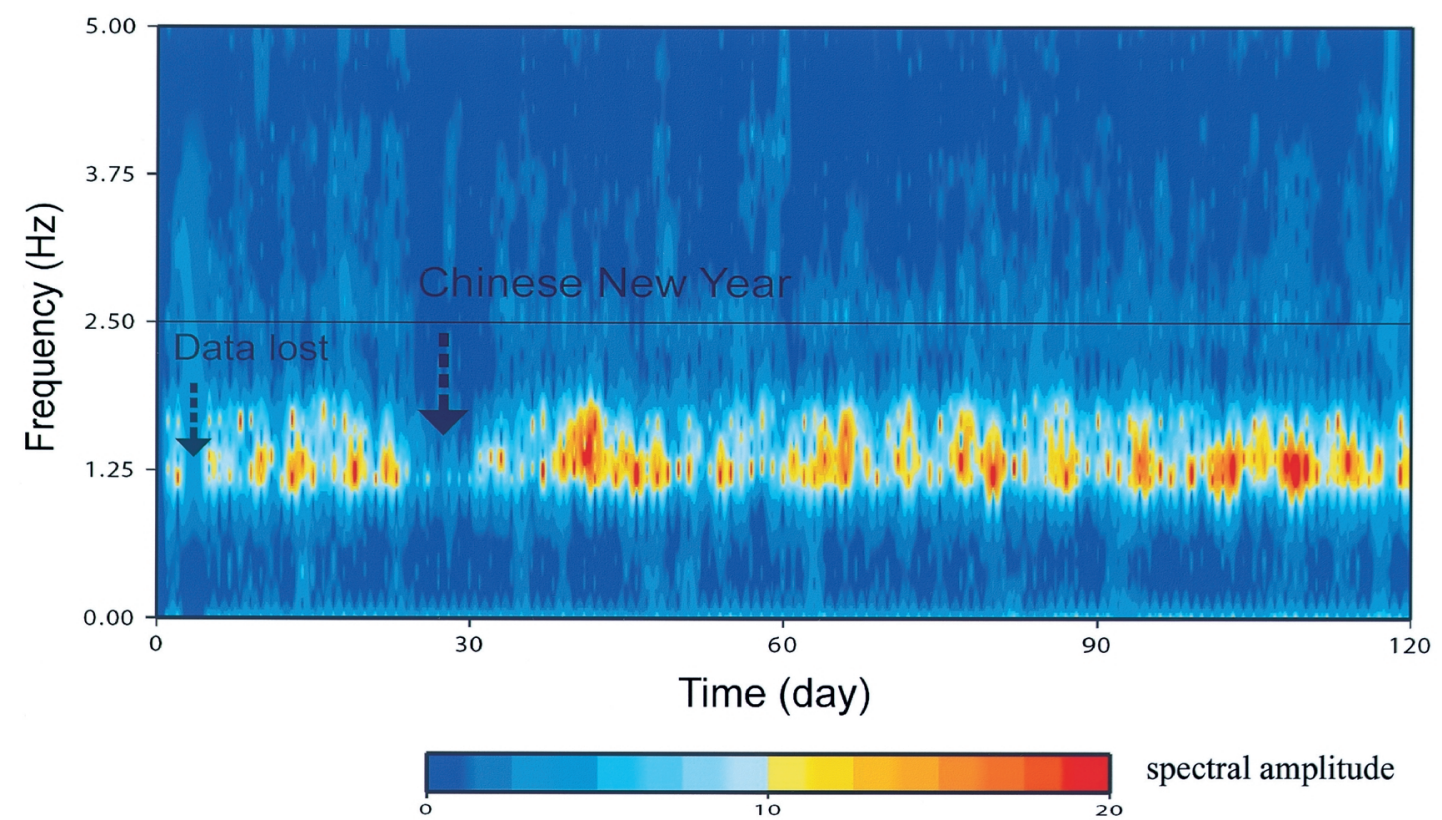

Fig. 8. The time-frequency spectrum of the background signals recorded at the TWD station, the 120 day analyzed time window. Some signals (marked as yellow and red) were found to persist over time. Two short noise-quiet windows of the background signals indicated in this figure are discussed in detail in the text.

\section{DISCUSSIONS AND CONCLUSIONS}

The signals detected in this study persisted over time in the narrow-band frequency. Detailed examination of the seismic spectra displayed strong band-limit signals in the frequencies from 1.3 to $1.7 \mathrm{~Hz}$ with dominant peaks at $1.3,1.4$, and $1.7 \mathrm{~Hz}$. The other minor peaks at the double frequencies of these major peaks, $2.6 \mathrm{~Hz}, 2.8 \mathrm{~Hz}$ and $3.4 \mathrm{~Hz}$, were also identified. The characteristics of double frequency picks, usually considered as an index of the artificial sources (Bokelmann and Baisch 1999), could not be found on the microtremors recorded by the CWBSN stations outside of the Hualien area.

Both the F-K results and the waveform stacking map showed a possible source location in the southwest portion of the survey area. The energy distributions of the arrays suggested predominant signals propagated from the southwest. The estimation based on the waveform stacking showed a significant high value region close to the J-array. Herein, we deduced that major narrow-band background noises came from the southwest of the survey area and maybe close to the J-array.

Unlike the signals incident from the southwest, the arrivals incident from other directions 
showed complex and incoherent results from individual arrays. Employing the F-K analyses for each array and the back-azmiths of the incident wave directions to estimate the sources, multiple candidate source locations were found. There were two possible interpretations for these sources in which those signals came from an individual energy source small enough to not be detected by all arrays. The other interpretation considered scattered sources near each array, and therefore signals were only recorded by the stations within a short distance and with difficulty in determining the precise location by all arrays.

In the Hualien area, the artificial sources could be induced by numerous plants using machine with fixed rotational frequencies. Based upon the inspection of the F-K and waveform stacking analyses, the observed signals were generated from large marble plants as a source of high amplitude microtremors. This assumption was sustained by the field investigation during array operation and especially in the south where many such plants operate.

Although all the spectral responses showed a primary energy level between $1.3 \sim 1.7 \mathrm{~Hz}$, the amplitude of the signals varied slightly within the arrays. Considering the possible source locations posited in this study, major signals propagate from south to north. Hence the recorded amplitudes of this narrow-band background noise would be smaller in the northern arrays than those in the southern arrays as shown in Fig. 4. Furthermore, the attenuation effect was also notable within the power spectra in which the relative amplitude at high frequency was smaller in the $\mathrm{N}$-array than those in the $\mathrm{J}$ and $\mathrm{K}$-arrays.

Furthermore, according to the F-K analyses, phase velocities of the background noises at each array were very slow leading to the inference that the signals propagated mostly through soft sediments. The determined phase velocity variation between arrays suggested that the thickness of the sediments below each was somewhat varied. The phase velocities of the incident waves from the eastern directions were usually smaller than those from the western directions due to thicker sediments in the eastern part of the survey area. The geological structure of the area is in concordance with this inference.

Based on the analyses of this study, the origin and characteristics of these seismic noises were clearly identified and that data may be useful to further isolate the background noise during future regional seismic observations and yield still higher quality seismic data.

Acknowledgements The authors wish to express their appreciation to the Central Weather Bureau and the Institute of Earth Sciences, Academia Sinica for providing data used in this study. They also thank Prof. K. L. Wen, C. Y. Wang and Dr. W. G. Huang for their useful comments and valuable discussions. This study was supported by National Central University, Academia Sinica and the National Science Council, R.O.C., under grants NSC90-2119-M001-005 and NSC90-2116-M-001-011.

\section{REFERENCES}

Aki. K., and P. G. Richards, 1980: Quantitative seismology: Theory and methods. Freeman, San Francisco, 932pp. 
Almendros, J., J. Ibanez, G. Alguacil, J. Morales, E. Del Pezzo, M. La Rocca, R. Ortiz, V. Arana, and M. J. Blanco, 2000: A double seismic antenna experiment at Teide Volcano: Existance of local seismicity and lack of evidences of volcanic tremor. J. Volcan. Geotherm. Res., 103, 439-462.

Almendros, J., B. Chouet, and P. Dawson, 2002: Array detection of a moving source. Seism. Res. Lett., 73, 153-165.

Asten, M. W., and J. D. Henstridge, 1984: Array estimators and the use of microseisms for reconnaissance of sedimentary basins. Geophys., 49, 1828-1837.

Bokelmann, H. R. G., and S. Baisch, 1999: Nature of narrow-band signals at $2.083 \mathrm{~Hz}$. Bull. Seism. Soc. Am., 89, 156-164.

Capon, J., 1969: High-resolution frequency-wavenumber spectrum analysis. Proc. IEEE., 57, 1408-1418.

Douze, E. J., 1967: Short-period seismic noise. Bull. Seism. Soc. Am., 57, 55- 81.

Haubrich, R. A., and K. McCamy, 1969: Microseisms; coastal and pelagic sources. Rev. Geophy., 7, 539-571.

Huang, W. G., and Y. T. Yeh, 1990: The characteristics of microtremors at the site of SMART1 array. Terr. Atmos. Ocean. Sci., 1, 225-242.

Lacoss, R. T., E. J. Kelly, and M. N. Toksoz, 1969: Estimation of seismic noise structure using arrays. Geophys., 34, 21-36.

Walker, R., J. Menard, and B. Bogert, 1964: Real-time high-resolution spectroscopy of seismic background noise. Bull. Seism. Soc. Am., 54, 501-509. 